\title{
Frontotemporal Dementia: The Impact of Patient Behavioral Symptoms on the Physical and Mental Health of Family Caregivers
}

\author{
Cindy C. Wong ${ }^{a}$ Margaret I. Wallhagen ${ }^{b}$ \\ a School of Nursing, Dominican University of California, San Rafael, Calif., and \\ ${ }^{b}$ School of Nursing, University of California San Francisco, San Francisco, Calif., USA
}

\section{Key Words}

Frontotemporal dementia - Patient behavioral symptoms • Caregiver physical health •

Caregiver mental health $\cdot$ Emotional distress

\begin{abstract}
Background: Providing informal support to someone with frontotemporal dementia (FTD) could be very stressful. Clarifying the relationship between patient behavioral problems and caregiver health could spur future research on effective symptom management strategies. Methods: Sixty-one FTD family caregivers participated in a postal survey. Results: Patient symptom severity was negatively associated with caregiver mental health $(r=-0.26, p<0.05)$ but not significantly associated with caregiver physical health. In a regression analysis, caregiver emotional distress from patient behaviors made a statistically significant contribution to caregiver mental health, explaining approximately $10 \%$ of its variance. Conclusion: This study underscores the importance of focusing on FTD caregivers' perceived emotional distress from patient behavioral problems and ensuring they are getting the appropriate support they need.
\end{abstract}

Copyright $\odot 2012$ S. Karger AG, Basel

\section{Introduction}

Frontotemporal dementia (FTD), a term that describes a group of conditions that involve the frontotemporal area of the brain, is a common cause of early-onset dementia, with a prevalence reported to be 81 per 100,000 [1]. It occurs in 5-15\% of individuals with dementia 
and frequently affects younger individuals between the ages of 45 and 65 years [2, 3]. Social awkwardness and behavioral changes, such as apathy and disinhibition, are common initial symptoms of FTD $[2,4]$. Patients with the behavioral variant type of FTD (bvFTD) usually present with a broad range of behavioral and neuropsychiatric symptoms, such as social avoidance, personal neglect, overeating, wandering, and an unusual preference for sweet foods and carbohydrates [5]. Because individuals with bvFTD usually exhibit severe and unusual behavioral disturbances, family caregivers can find it particularly challenging and overwhelming to provide care for someone with this form of dementia.

Prior research studies have shown that the behavioral disturbances common in patients with bvFTD are strong contributors to caregiver burden and psychological distress [6-8]. For instance, in their study of the impact of behavioral functioning in dementia on caregiver burden ( $\mathrm{n}=72$ ), Davis and Tremont [9] found that behaviors related to disinhibition and executive dysfunction were predictors of caregiver burden $(t=2.09, \beta=0.36, \mathrm{p}<0.01)$. Mourik et al. [10] studied which behavioral symptoms occur together in clusters $(n=63)$ and found the following two clusters: (1) mood (comprising anxiety and depression), and (2) agitation/ psychosis (comprising agitation, delusions, hallucinations, and irritability). The researchers also investigated the relationship between behavioral clusters and caregiver distress $(\mathrm{n}=$ 63) [10]. After controlling for confounding variables, they found caregiver distress to be strongly related to agitation/psychosis $(b=0.56, \mathrm{p}<0.0001)$, followed by mood $(b=0.27$, $\mathrm{p}<0.001)[10]$.

Conversely, in their 2-year longitudinal study, Riedijk et al. [11] found that caregiver emotional burden caused by patient neuropsychiatric symptoms significantly decreased $(\mathrm{n}=63 ; \mathrm{p}<0.01)$ from baseline (mean $\pm \mathrm{SD}=9.4 \pm 6.8)$ to 24 months $(6.4 \pm 4.9)$. Overall, caregiver burden also decreased significantly $(\mathrm{p}<0.01)$ during the 2 years (baseline: $5.6 \pm$ 2.6; 24 months: $4.2 \pm 2.8$ ). However, selective dropout is a potential limitation because 10 dyads who dropped out for unknown reasons had higher scores on patient neuropsychiatric disturbance and subsequent caregiver burden [11]. Nevertheless, these findings support the hypothesis that the negative effect of patient neuropsychiatric symptoms on family caregivers may decrease over time as caregivers become more accustomed to their role and develop better strategies for managing patient neuropsychiatric and/or behavioral symptoms.

Despite these findings, few research studies have been conducted on the impact of patient symptoms on the physical and mental health of FTD family caregivers. In the United States, the vast majority of adults (78\%) who receive long-term care at home get that care from family caregivers; unfortunately, many family caregivers experience deteriorating health as a result of their efforts [12-14]. Furthermore, family caregivers of persons with dementia have reported more physical and mental health problems as a result of their caregiving than caregivers of non-demented persons $[15,16]$. This finding underscores the importance of focusing on factors that affect the health and well-being of FTD family caregivers. Clarifying the relationship between patient behavioral problems and the health of FTD family caregivers could spur future research on effective strategies for managing unusual behavioral problems common in patients with FTD. Also, if health-care professionals are to develop a fuller appreciation of the experiences of FTD family caregivers, they must understand the different types of patient behavioral problems that these family caregivers routinely manage.

The aims of this descriptive, correlational study were: (1) to examine the relationship between the severity of behavioral problems in FTD patients and the physical and mental health of their family caregivers; (2) to describe the frequency of behavioral problems in patients with FTD and their emotional impact on family caregiver, and (3) to assess the relationship between caregiver emotional distress from patient behavioral problems and caregiver physical and mental health. 
This study is unique in specifically assessing the frequency of distinctive behaviors commonly manifested in bvFTD, such as social avoidance, criminal behavior like shoplifting, and perseverative/obsessive behaviors.

\section{Methods}

\section{Participants}

In order to identify family caregivers of persons with FTD and exhibiting behavioral problems, support groups which were associated with clinics that did comprehensive dementia evaluations as well as the Association for Frontotemporal Degeneration (AFTD) were utilized for recruitment. Specifically, potential participants were recruited from FTD caregiver support group meetings in Portland, Oreg., and San Francisco, Calif., and information on the study was included in the newsletters and on the website (http://www.theaftd.org/) of the AFTD. To be included in the study, caregivers had (a) to identify themselves as the family member who is primarily responsible for the patient's care; (b) to have provided care to the patient for at least 6 months; (c) to live with the patient; (d) to receive no financial compensation for caregiving; (e) to speak, read, and understand English, and (f) to be 18 years of age or older. If more than one caregiver was involved in the patient's care, the family caregiver providing most of the care was selected. The eligibility criterion for the patients was a diagnosis of FTD with behavioral problems, as reported by his or her caregiver. Recruitment occurred between June 2011 and November 2011.

Interested and eligible family caregivers completed a set of questionnaires that took roughly $30 \mathrm{~min}$ to complete. Upon receipt of the questionnaires, the participants had the option of receiving a USD 5 Safeway gift card as an acknowledgement of their time. Completed questionnaires were returned anonymously by standard mail.

Human subjects approval was obtained from the University of California San Francisco's Committee on Human Research. In an introductory letter, the participants were notified that consent would be implied if the caregiver completed and submitted the survey. All participants were provided with an informed consent document which provided additional information on the study, such as the study's purpose, procedures, risks, and benefits.

Data from 61 caregivers were collected and analyzed. The demographic characteristics of the caregivers and patients included age, gender, and race/ethnicity. Caregivers were also asked about their relationship to the patient (e.g., spouse, child, or sibling), their marital status (e.g., single, married, or partnered), the number of years they had known the patient, the number of years they had been providing care to the patient, and additional paid and unpaid sources of support.

\section{Instruments}

Caregiver Physical and Mental Health

The participants' physical and mental health status was assessed by the physical and mental health component scores from the 12-Item Short-Form Health Survey (SF-12) [17]. The SF-12, which was derived from the 36-Item Short-Form Health Survey, is a generic measure of health that can be used in different settings $[18,19]$. The SF-12 can be self-administered or given in person by a trained interviewer [17]. Test-retest (2-week) correlations for the Physical Component Summary (PCS) and the Mental Component Summary (MCS) have been found to be 0.89 and 0.76 , respectively, in the general US population $(\mathrm{n}=232)$ [17]. Furthermore, the SF-12 has been shown to produce two summary scales (physical health and mental health), originally developed from the SF-36, which offer good accuracy and decreased respondent burden [20]. 
Table 1. Additional NPI-Q items

1 Loss of insight: During the past month, was the patient unaware of any problems and/or changes in his/her behavior?

2 Social inappropriateness: During the past month, did the patient say and/or do things that are socially unacceptable? This may include being rude or acting childish.

3 Social avoidance: During the past month, did the patient seem socially disengaged and/or avoided social situations and interactions with others?

4 Criminal behavior: During the past month, was the patient involved in any type of criminal behavior, such as traffic violations, shoplifting, and public indecency?

5 Hypersexuality: During the past month, has the patient's sexual behavior been unusually excessive? This may include making sexual remarks, touching others inappropriately, and undressing more frequently.

6 Hyposexuality: During the past month, has the patient's sexual behavior been unusually diminished or absent? This may include difficulty achieving sexual arousal and/or an inability to achieve an orgasm.

7 Preference for sweets: During the past month, has the patient been consuming excessive quantities of sweets, such as candies and pastries?

8 Perseverations/obsessions: During the past month, has the patient been repeating actions and/or remarks?

Patient Neuropsychiatric Symptoms

The Neuropsychiatric Inventory Questionnaire (NPI-Q) [21, 22] was used to assess patient behavioral problems. The NPI-Q assesses the following 12 neuropsychiatric disturbances common in dementia patients: aberrant motor behavior, agitation, anxiety, apathy, appetite and eating disorders, delusions, disinhibition, dysphoria/depression, euphoria, hallucinations, irritability, and nighttime behavior disturbances. The NPI-Q was cross-validated with the standard NPI $[23,24]$ to provide a quantitative measure of patient symptom severity and caregiver distress in relation to different neuropsychiatric disturbances [21]. The total symptom severity score ranges between 0 and 36; higher scores reflect greater severity of patient neuropsychiatric symptoms. The total caregiver distress score ranges between 0 and 60; higher scores reflect greater caregiver distress in relation to patient neuropsychiatric symptoms. The reliability and validity of this measure have been previously established [21,22].

Because the NPI-Q does not assess some behaviors that are common in patients with bvFTD, we developed and included the following 8 additional NPI-Q items to assess these symptoms/behavioral disturbances: loss of insight, social inappropriateness, social avoidance, criminal behavior, hypersexuality, hyposexuality, preference for sweets, and perseverations/obsessions. The study investigator (C.C.W.) verified these items with family caregivers at a FTD caregiver support group meeting in San Francisco. For these 8 additional NPI-Q items, each question corresponded to a symptom/behavioral problem (table 1). Participants were first asked to respond Yes (present) or No (absent) to each question. If caregivers answered No, they proceeded to the next question. If they answered Yes, they rated the severity of symptoms in the previous month on a 3-point scale. Participants were then asked to rate the amount of emotional distress caused by each neuropsychiatric symptom on a 6-point scale. The total symptom severity score for the 8 additional NPI-Q items ranges between 0 and 24; higher scores reflect greater severity of patient neuropsychiatric symptoms. The total caregiver distress score ranges between 0 and 40; higher scores reflect greater caregiver distress in relation to patient neuropsychiatric symptoms. 
Table 2. Caregiver and patient demographic characteristics, NPI-Q, MCS, and PCS

\begin{tabular}{lcc}
\hline & Caregivers $(\mathrm{n}=61)$ & Patients $(\mathrm{n}=61)$ \\
\hline Gender & & \\
$\quad$ Female & $48(78.7)$ & $20(32.8)$ \\
$\quad$ Male & $13(21.3)$ & $41(67.2)$ \\
Age, years & $61.85 \pm 10.83$ & $65.79 \pm 9.35$ \\
Relationship to patient & $55(90.2)$ & \\
$\quad$ Spouse (or equivalent) & $4(6.6)$ & \\
$\quad$ Daughter & $2(3.3)$ & \\
$\quad$ Other & & $1(1.6)$ \\
Race/ethnicity & $57(93.4)$ & $1(1.6)$ \\
$\quad$ Caucasian/White & $1(1.6)$ & \\
$\quad$ African-American/Black & $1(1.6)$ & \\
$\quad$ Hispanic & $2(3.7)$ & $12.45 \pm 2.49$ \\
$\quad$ Chinese & $39.39 \pm 13.83$ & \\
Duration caregiver has known patient, years & $5.96 \pm 8.03$ & \\
Duration of caregiving, years & & \\
Number of years since diagnosis & & \\
NPI-Q Severity score $(0-36, \mathrm{n}=59)$ & $15.36 \pm 8.52$ & \\
NPI-Q Distress score $(0-60, \mathrm{n}=58)$ & $51.49 \pm 11.22$ & \\
PCS score & $42.49 \pm 10.96$ & \\
MCS score &
\end{tabular}

Results presented as means \pm SD or $\mathrm{n}(\%)$.

Data Analysis

We used SPSS version 19.0 to conduct all data analyses. For the quantitative variables of interest, mean scores were calculated to assess central tendency along with their standard deviations (SD) as a measure of dispersion. To assess the magnitude and direction of the linear relationships between the quantitative variables of interest, Pearson's correlation coefficient tests were conducted.

After examining bivariate relationships, two separate standard multiple linear regression tests were conducted to determine whether patient symptom severity and caregiver distress from patient symptoms made statistically significant unique contributions to caregiver mental health above and beyond the influence of other important variables (e.g., caregiver age). The selection of the predictors was based on preliminary analyses of the associations between the quantitative variables of interest and the study's aims. Preliminary analyses were also conducted to ensure no violation of the assumptions of linearity, normality, multicollinearity, and homoscedasticity occurred. Multiple regression analyses that violated these assumptions were not reported. Because there are no prior studies on the reliability and validity of the NPI-Q items that were added, the scores on this component of the study were not included in the multiple regression analyses.

For the first multiple regression (MR 1), the dependent variable was caregiver mental health, and the independent variables entered into the model were the NPI-Q Severity scores (total patient symptom severity score from the NPI-Q), caregiver age, the number of years caregivers had known their patient, and the number of years they had been providing care to that person. For the second multiple regression (MR 2), the dependent variable was still caregiver mental health, but the independent variables entered into the model were the NPIQ Distress scores (total caregiver distress score from the NPI-Q), caregiver age, the number of years caregivers had known their patient, and the number of years they had been provid- 
Table 3. NPI-Q and the 8 additional neuropsychiatric symptoms

\begin{tabular}{llll}
\hline Symptom & $\begin{array}{l}\text { Frequency } \\
\mathrm{n}(\%)\end{array}$ & $\begin{array}{l}\text { Patient symptom severity } \\
\text { mean } \pm \text { SD (median) }\end{array}$ & $\begin{array}{l}\text { Caregiver distress } \\
\text { mean } \pm \text { SD (median) }\end{array}$ \\
\hline NPI-Q & & & \\
Apathy/indifference & $51(83.6)$ & $1.82 \pm 1.03(2)$ & $2.17 \pm 1.53(2)$ \\
Appetite/eating problems & $46(75.4)$ & $1.56 \pm 1.15(2)$ & $1.65 \pm 1.55(1)$ \\
Disinhibition & $39(63.9)$ & $1.31 \pm 1.15(1)$ & $1.62 \pm 1.65(1)$ \\
Agitation/aggression & $38(62.3)$ & $1.08 \pm 1.04(1)$ & $1.57 \pm 1.54(1)$ \\
Motor disturbance & $36(59)$ & $1.18 \pm 1.13(1)$ & $1.38 \pm 1.50(1)$ \\
Anxiety & $36(59)$ & $1.05 \pm 1.06(1)$ & $1.48 \pm 1.52(1)$ \\
Irritability & $34(55.7)$ & $1.03 \pm 1.08(1)$ & $1.23 \pm 1.46(1)$ \\
Nighttime behaviors & $32(52.5)$ & $1.07 \pm 1.16(1)$ & $0.77 \pm 1.28(0)$ \\
Depression/dysphoria & $22(36.1)$ & $0.61 \pm 0.88(0)$ & $0.69 \pm 1.23(0)$ \\
Delusions & $20(32.8)$ & $0.54 \pm 0.89(0)$ & $0.46 \pm 1.09(0)$ \\
Elation/euphoria & $15(24.6)$ & $0.49 \pm 0.94(0)$ & $0.43 \pm 0.94(0)$ \\
Hallucinations & $15(24.6)$ & $0.41 \pm 0.82(0)$ & $1.82 \pm 1.46(2)$ \\
Additional NPI-Q items & & & $1.41 \pm 1.41(1)$ \\
Loss of insight & $46(75.4)$ & $1.18 \pm 0.92(1)$ & $1.34 \pm 1.53(1)$ \\
Social inappropriateness & $41(67.2)$ & $1.02 \pm 0.90(1)$ & $1.30 \pm 1.35(1)$ \\
Perseverations/obsessions & $39(63.9)$ & $1.02 \pm 0.98(1)$ & $0.85 \pm 1.30(0)$ \\
Social avoidance & $37(60.7)$ & $0.98 \pm 0.96(1)$ & $0.49 \pm 1.15(0)$ \\
Preference for sweets & $28(45.9)$ & $0.74 \pm 0.98(0)$ & $0.43 \pm 1.15(0)$ \\
Hyposexuality & $17(27.9)$ & $0.39 \pm 0.76(0)$ & $0.31 \pm 1.07(0)$ \\
Hypersexuality & $9(14.8)$ & $0.30 \pm 0.78(0)$ & \\
Criminal behavior & $6(9.8)$ & $0.20 \pm 0.68(0)$ & \\
\hline
\end{tabular}

ing care to that person. Although the maximum Mahalanobis distance value of 25.52 for MR 1 and 26.6 for MR 2 suggests the presence of outliers, the maximum Cook distance value for both analyses was less than 1 , indicating that no outliers were having an undue influence on the results of the models [25].

According to Hulley et al. [26], the sample size requirements when using the correlation coefficient $(r)$ for a medium effect size would be 85 subjects $(\beta=0.20, \alpha=0.05$, two-sided test). Due to the lack of previous FTD caregiving studies on this particular topic, the selection of a medium effect size was based on previous dementia caregiving studies assessing the association between patient behavioral disturbances/neuropsychiatric symptoms and caregiver burden [6]. In addition, according to Tabachnick and Fidell [25], a sample size of 82 would be required to run a multiple regression with 4 independent variables $[50+(8 \times 4)=$ 82]. To account for potential missing data, however, the target sample size was 95 caregivers. Alpha was set at 0.05 for all data analyses.

\section{Results}

Caregiver and Patient Demographic Characteristics

Table 2 presents the demographic characteristics of the sample. The caregivers had an average age of 62 years (range 35-90), and most were women (78.7\%), Caucasian/White (93.4\%), and married or partnered (95.1\%). They had known their patient for an average of 39 years and had been providing care for an average of 6 years. About half (52.5\%) of the caregivers were not receiving any type of paid support at home; $43 \%$ were receiving some type of paid support 1-2 days per week. Almost half (47.5\%) of the caregivers were receiving 
Fig. 1. Frequency of each neuropsychiatric symptom from the NPI-Q.

Fig. 2. Frequency of the 8 additional neuropsychiatric symptoms.
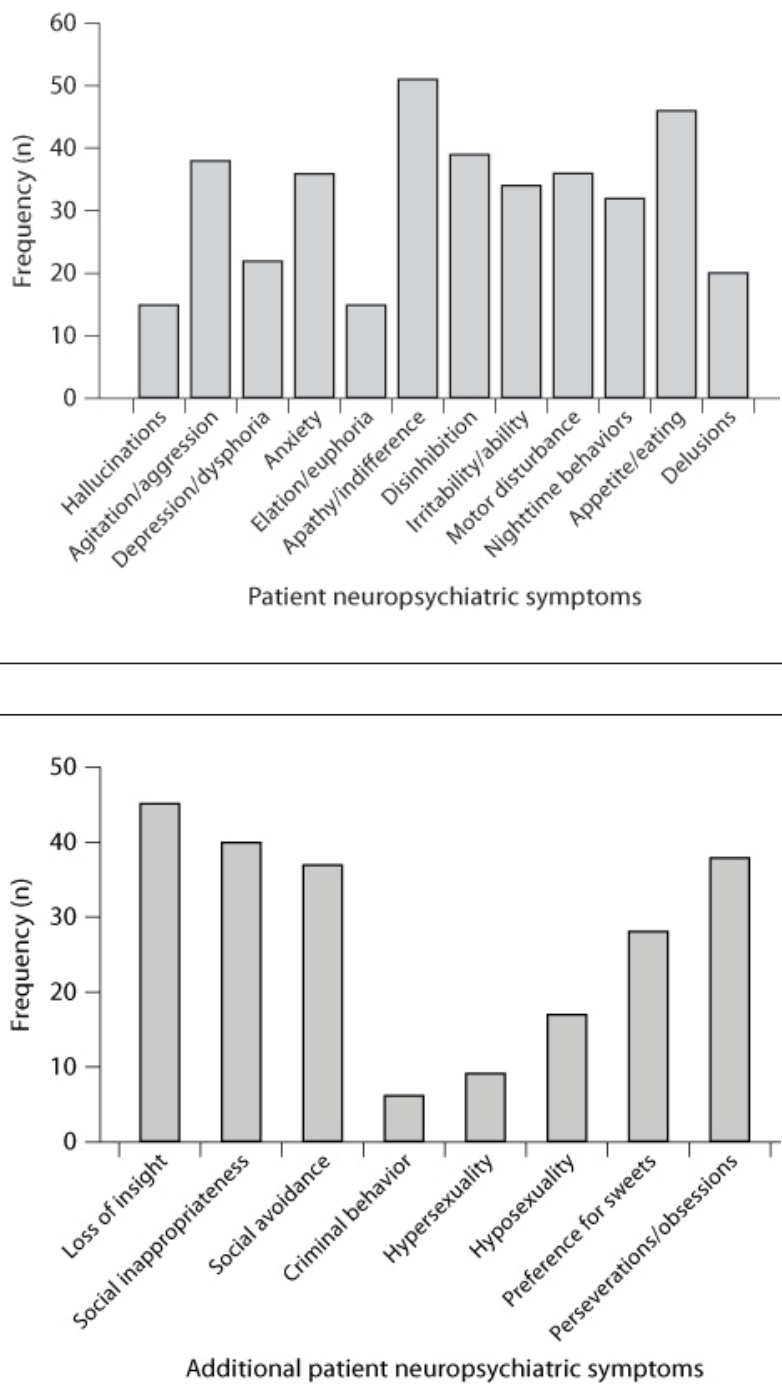

no unpaid support from friends or relatives; about $44 \%$ of the caregivers were receiving unpaid support 1-2 days a week.

The average age of the patients was 66 years (range 38-88), and the average number of years since FTD diagnosis was about $4(\mathrm{SD}=2.49)$. Most patients were men $(67.2 \%)$ and Caucasian/White (95.1\%).

\section{Patient Neuropsychiatric Symptoms and Behavioral Problems}

Table 3 presents the frequency, means, and SD of each neuropsychiatric symptom on the NPI-Q and the 8 additional neuropsychiatric symptoms. Figures 1 and 2 illustrate the number (frequency) of patients who exhibited each neuropsychiatric symptom. Eighty-four percent of the patients exhibited some degree of apathy/indifference. Other common neuropsychiatric symptoms included loss of insight (75\%), appetite/eating problems (75\%), social inappropriateness (67\%), perseverations/obsessions (64\%), and disinhibition (64\%). Caregivers were most distressed when their patient exhibited apathy/indifference (mean $\pm \mathrm{SD}=2.17$ 
Wong et al.: Frontotemporal Dementia: The Impact of Patient Behavioral Symptoms on the Physical and Mental Health of Family Caregivers

Table 4. Correlations between self-reported measures: NPI-Q, additional NPI-Q items, MCS, and PCS

\begin{tabular}{|c|c|c|c|c|c|c|c|}
\hline & Measure: & 1 & 2 & 3 & 4 & 5 & 6 \\
\hline 1 & NPI-Q Severity & 1 & & & & & \\
\hline 2 & NPI-Q Distress & $0.86^{*}$ & 1 & & & & \\
\hline 3 & Additional NPI-Q Severity & $0.71^{*}$ & $0.67^{*}$ & 1 & & & \\
\hline 4 & Additional NPI-Q Distress & $0.58^{*}$ & $0.66^{*}$ & $0.86^{*}$ & 1 & & \\
\hline 5 & MCS & $-0.26^{*}$ & $-0.40^{*}$ & $-0.34^{*}$ & $-0.36^{*}$ & 1 & \\
\hline 6 & PCS & -0.06 & -0.07 & 0.02 & -0.01 & $-0.26^{*}$ & 1 \\
\hline
\end{tabular}

$\pm 1.53)$ and loss of insight $(1.82 \pm 1.46)$. Less commonly reported symptoms included elation/euphoria (25\%), hallucinations (25\%), hypersexuality (15\%), and criminal behavior (10\%).

Correlations between Patient Neuropsychiatric Symptoms and Caregiver Health

Table 4 presents the correlations between the NPI-Q Severity scores, NPI-Q Distress scores, Additional NPI-Q Severity scores (total symptom severity score for the 8 additional neuropsychiatric symptoms), Additional NPI-Q Distress scores (total caregiver distress score for the 8 additional neuropsychiatric symptoms), MCS scores (as a measure of caregiver mental health), and PCS scores (as a measure of caregiver physical health). As expected, moderate-to-strong, statistically significant positive relationships were observed between the NPIQ Severity scores, NPI-Q Distress scores, Additional NPI-Q Severity scores, and Additional NPI-Q Distress scores ( $\mathrm{p}>0.05$ for all). The NPI-Q Severity scores had a small, statistically significant negative association with the MCS scores $(r=-0.26, \mathrm{p}<0.05)$. Also, a small, statistically significant negative relationship was found between the Additional NPI-Q Severity scores and the MCS scores $(r=-0.34, \mathrm{p}<0.05)$ and between the Additional NPI-Q Distress scores and the MCS scores $(r=-0.36, \mathrm{p}<0.05)$. A medium negative relationship was noted between the NPI-Q Distress scores and the MCS scores $(r=-0.40, \mathrm{p}<0.05)$. The severity of patient symptoms (as measured by the NPI-Q and Additional NPI-Q Severity scores) and caregiver distress from patient symptoms (as measured by the NPI-Q Distress and Additional NPI-Q Distress scores) were not statistically significantly related to the PCS scores ( $p>0.05$ for all). Standard regression analyses were subsequently conducted to determine whether the NPI-Q Severity scores and the NPI-Q Distress scores both made statistically significant unique contributions to caregiver mental health above and beyond the influence of other potentially influential variables.

\section{Multiple Regression Analyses}

MR 1 was conducted to assess the association between the NPI-Q Severity scores and the MCS scores beyond the influence of caregiver age, the number of years a caregiver had known the patient, and the number of years he or she had been providing care to the patient. Approximately $34.5 \%$ of the variance was explained by the model with 4 independent variables, and the entire model was statistically significant $\left(R^{2}=0.35, F(4,54)=7.11, \mathrm{p}<0.05\right)$. Only caregiver age made a statistically significant unique contribution to the MCS scores $(B$ $[\mathrm{SE}]=0.40[0.15], \beta=0.40, \mathrm{p}<0.05)$ and explained approximately $9 \%$ of the variability in the MCS scores. The NPI-Q Severity scores did not make a statistically significant contribution to the MCS scores $(B[\mathrm{SE}]=-0.24[0.21], \beta=-0.14, \mathrm{p}=0.25)$ and only explained about 
$1.6 \%$ of the unique variance in the MCS scores. The number of years a caregiver had known the patient $(B[\mathrm{SE}]=0.12[0.11], \beta=0.16, \mathrm{p}=0.28)$ and the number of years the caregiver had been providing care to the patient $(B[\mathrm{SE}]=0.16[0.15], \beta=0.12, \mathrm{p}=0.30)$ did not make a statistically significant unique contribution to the MCS scores.

MR 2 was conducted to determine whether the NPI-Q Distress scores made a statistically significant unique contribution to the MCS scores above and beyond the influence of caregiver age and how long a caregiver had known and had been providing care to the patient. About $42.3 \%$ of the variance was explained by the model with 4 independent variables, and the entire model was statistically significant $\left(R^{2}=0.43, F(4,53)=9.72, \mathrm{p}<0.05\right)$. The NPI-Q Distress scores made a statistically significant unique contribution to the MCS scores $(B[\mathrm{SE}]=-0.41[0.14], \beta=-0.32, \mathrm{p}<0.05)$ and explained approximately $9.5 \%$ of the variability in the MCS scores. Caregiver age also made a statistically significant unique contribution to the MCS scores $(B[\mathrm{SE}]=0.34[0.14], \beta=0.34, \mathrm{p}<0.05)$ and explained approximately $6.6 \%$ of the variability in the MCS scores. The number of years a caregiver had known the patient $(B[\mathrm{SE}]=0.15[0.11], \beta=0.19, \mathrm{p}=0.17)$ and the number of years the caregiver had provided care to the patient $(B[\mathrm{SE}]=0.17[0.15], \beta=0.13, \mathrm{p}=0.24)$ did not make statistically significant unique contributions to the MCS scores.

\section{Discussion}

The purpose of this study was to describe the frequency of different behavioral problems exhibited by patients with FTD and the emotional impact of these behaviors on family caregivers. This study also examined the relationship between caregiver health and patient symptom severity in addition to caregiver emotional distress from patient symptoms. The most frequently reported patient symptoms included apathy/indifference (84\%), loss of insight (75\%), appetite/eating problems (75\%), and social inappropriateness (67\%). Caregivers reported the greatest emotional distress from patient apathy/indifference and loss of insight. Even so, their average score were still only registered as 'mildly distressed'.

Many of our caregivers were participating in support groups and/or belonged to an association that supports caregivers of persons with FTD. Thus, these individuals may have had greater social support networks than other caregivers who did not have such support. In fact, more than $40 \%$ of the caregivers were receiving some type of paid support at least 1-2 days a week (e.g., paid formal caregiver) and unpaid support at least 1-2 days a week from friends, relatives, or neighbors. Thus, a number of the family caregivers in this study may have learned to effectively manage patient behaviors to minimize their negative emotional impact. This could partially explain why the participants, on average, were only mildly distressed by patient apathy/indifference and minimally-to-mildly distressed by patient agitation/aggression, appetite and eating problems, disinhibition, and loss of insight.

An inverse relationship was observed between the severity of patient behavioral problems and caregiver mental health, suggesting that less severe behavioral disturbances are associated with better caregiver mental health. However, in a multiple regression analysis, patient symptom severity did not make a statistically significant unique contribution to caregiver mental health after controlling for variables such as caregiver age and duration of caregiving. Consequently, other situational and demographic factors must be considered when examining the relationship between patient symptom severity and caregiver mental health. Our results suggest that the negative effect of patient symptom severity on family caregivers may be mitigated by other personal and environmental factors, such as the caregivers' previous caregiving experiences and available resources. Future research is needed to 
Wong et al.: Frontotemporal Dementia: The Impact of Patient Behavioral Symptoms on the Physical and Mental Health of Family Caregivers

identify potential variables that mediate or moderate the relationship between the severity of patient neuropsychiatric symptoms and caregiver health and well-being.

On the other hand, caregiver emotional distress from patient behavioral problems was negatively associated with caregiver mental health and made a statistically significant unique contribution to caregiver mental health above and beyond the influence of the caregivers' age and how long they had known and been providing care to their patient in the multiple regression analyses. This finding suggests that the caregivers' personal experiences and perceived emotional distress from patient behaviors may be more important and influential to caregiver mental health than merely the severity of their patient's behavioral problems. Despite the severity of a patient's behavioral problems, caregiver mental health may not be as affected or jeopardized if caregivers do not perceive these behaviors to be particularly distressing and unmanageable. In contrast, no statistically significant relationship was detected between the severity of patient behavioral disturbances (in addition to caregiver distress from patient behaviors) and caregiver physical health.

The results of this study provide additional support for the findings of previous studies that examined the effect of patient behavioral problems on the experiences of FTD family caregivers. For instance, De Vugt et al. [27] compared caregiver emotional distress from patient behavioral symptoms between caregivers of patients with Alzheimer's disease and FTD and found that apathy was the most common behavioral symptom and occurred more often among FTD patients (89\%). In addition, the most distressing symptom for caregivers of FTD patients was apathy (mean $\pm \mathrm{SD}=2.4 \pm 1.6)$ followed by disinhibition $(2.0 \pm 1.7)$ [27]. In Mourik et al.'s [10] study, apathy was noted in $95 \%$ of patients, with a mean composite score of 8.9 ( $\mathrm{SD}=3.3)$. Apathy has been defined as a disorder of diminished motivation that may cause clinically significant impairment in one's social and personal functioning [28]. In patients with FTD, apathy has been associated with caregiver emotional distress in addition to increased immobility and lower levels of activity [29]. When a patient becomes indifferent and is no longer interested in matters of concern to a caregiver, the caregiver may feel distressed by the perceived lack of an emotional connection to the patient as well as the loss of companionship and shared meanings.

We are unsure why there was a statistically significant negative relationship between caregiver mental and physical health; the explanation could be the mere characteristics and nature of the sample. The caregivers in this study who reported better mental health may also be experiencing more physical health problems due to older age or other unknown reasons. Further study is needed to investigate potential mediators and moderators influencing the relationship between caregiver physical and mental health in this population.

Individuals with bvFTD often present with a wide range of behavioral disturbances that are not typically seen in patients with Alzheimer's disease, such as personality changes, social awkwardness, and social inappropriateness [5]. Thus, acknowledging these behavioral problems and the emotional impact they have on FTD family caregivers is very important. As expected, there were moderate-to-large positive correlations between the NPI-Q scores and the scores for the 8 additional NPI-Q items in assessing both the severity of patient behavioral problems and caregiver distress from patient behaviors. All of the behavioral symptoms from the additional NPI-Q items (loss of insight, social inappropriateness, social avoidance, criminal behavior, hypersexuality, hyposexuality, preference for sweets, and perseverations/ obsessions) were seen in the patients. Seventy-five percent of the patients showed some degree of loss of insight, and more than half of the patients exhibited social inappropriateness (67\%), perseverative/obsessive behaviors (64\%), and social avoidance (61\%). These findings underscore the potential value of incorporating the 8 additional NPI-Q items into the NPI-Q when assessing patient behavioral problems and caregiver distress from patient behavioral disturbances in FTD. 
This study has a number of limitations that warrant consideration. First, the criteria used for establishing the FTD diagnosis were unknown because our survey was anonymous and the diagnosis of FTD was based on caregiver self-reports. However, the participants were contacted through an association that specifically supports caregivers of persons with FTD. Second, the sample size $(n=61)$ was relatively small. However, despite this small sample size, we still found statistically significant relationships between some of the quantitative variables of interest (e.g., the relationship between the severity of patient neuropsychiatric symptoms and caregiver mental health).

Despite its limitations, the current study is innovative. It focused on an understudied atrisk population of family caregivers of persons with FTD and examined the relationship between patient behavioral disturbances and caregiver health. Previous studies of dementia caregiving have not focused on the effect of patient behavioral disturbances on the physical and mental health of FTD family caregivers. Additional neuropsychiatric symptoms and/or behavioral disturbances not assessed by the NPI-Q but commonly seen in patients with bvFTD, such as loss of insight, hyposexuality, and preference for sweet foods, were also uniquely included. Furthermore, because the caregivers were recruited through the AFTD, a national organization, participants included caregivers living in various locations throughout the United States and Canada.

The findings of this study have important implications for clinicians and future research. They enlighten clinicians about the different behavioral problems that family caregivers of persons with FTD deal with and enable them to help such caregivers anticipate these behavioral problems before they occur. The negative relationship found between the severity of patient behavioral problems and caregiver mental health reinforces how important it is for clinicians to educate FTD family caregivers about strategies that can help them manage the severe behavioral disturbances common in bvFTD patients, thereby promoting caregiver mental health and well-being. When clinicians design caregiver interventions, this study's findings can help them focus on behavioral problems, such as apathy/indifference and loss of insight, which can be particularly distressing for family caregivers. Future caregiving studies are needed to assess the psychometric properties of the NPI-Q with the 8 additional NPI-Q items in a larger sample of FTD family caregivers and to examine the effectiveness of different symptom management strategies that family caregivers can use to effectively manage the behavioral disturbances that are commonly seen in patients with bvFTD. Additional longitudinal studies are also needed to understand the effect of specific patient behavioral problems on the health and well-being of FTD family caregivers over time.

\section{Acknowledgements}

We would like to thank Steve Paul, $\mathrm{PhD}$, for his assistance with the statistical analyses, the Association for Frontotemporal Degeneration for its assistance with recruitment, and Jennifer Merrilees, RN, $\mathrm{PhD}$, and Robin Ketelle, RN, MS, for their help with the study. We are also extremely grateful to all caregivers who participated in this study.

\section{Disclosure Statement}

The authors declare no conflicting interests with respect to authorship and/or the publication of this article. 


\section{References}

1 Ratnavalli E, Brayne C, Dawson K, Hodges JR: The prevalence of frontotemporal dementia. Neurology 2002;58:1615-1621.

-2 Mohandas E, Rajmohan V: Frontotemporal dementia: an updated overview. Indian J Psychiatry 2009;51:65-69.

-3 Weder ND, Aziz R, Wilkins K, Tampi RR: Frontotemporal dementias: a review. Ann Gen Psychiatry 2007;6:15-25.

-4 Lindau M, Almkvist O, Kushi J, Boone K, Johansson SE, Wahlund LO, Cummings JL, Miller BL: First symptoms - frontotemporal dementia versus Alzheimer's disease. Dement Geriatr Cogn Disord 2000;11:286-293.

-5 Snowden JS, Bathgate D, Varma A, Blackshaw A, Gibbons ZC, Neary D: Distinct behavioral profiles in frontotemporal dementia and semantic dementia. J Neurol Neurosurg Psychiatry 2001;70:323332.

-6 Knutson KM, Zamboni G, Tierney MC, Grafman J: Neural correlates of caregiver burden in cortical basal syndrome and frontotemporal dementia. Dement Geriatr Cogn Disord 2008;26:467-474.

7 Kumamoto K, Arai Y, Hashimoto N, Ideda M, Mizuno Y, Washio M: Problems family caregivers encounter in home care of patients with frontotemporal lobar degeneration. Psychogeriatrics 2004;4: 33-39.

8 Pinquart M, Sorensen S: Associations of stressors and uplifts of caregiving with caregiver burden and depressive mood: a meta-analysis. J Gerontol B Psychol Sci Soc Sci 2003;58:P112-P128.

-9 Davis DD, Tremont G: Impact of frontal systems behavioral functioning in dementia on caregiver burden. J Neuropsychiatry Clin Neurosci 2007;19:43-49.

-10 Mourik JC, Rosso SM, Niermeijer MF, Duivenvoorden HJ, van Swieten JC, Tibben A: Frontotemporal dementia: behavioral symptoms and caregiver distress. Dement Geriatr Cogn Disord 2004;18: 299-306.

-11 Riedijk S, Duivenvoorden H, Rosso S, Van Swieten J, Niermeijer M, Tibben A: Frontotemporal dementia: change of familial caregiver burden and partner relation in a Dutch cohort of 63 patients. Dement Geriatr Cogn Disord 2008;26:398-406.

-12 Barrow S, Harrison RA: Unsung heroes who put their lives at risk? Informal caring, health and neighbourhood attachment. J Public Health (Oxf) 2005;27:292-297.

-13 Lee S, Colditz GA, Berkman LF, Kawachi I: Caregiving and risk of coronary heart disease in U.S. women: a prospective study. Am J Prev Med 2003;24:113-119.

14 Thompson L: Long-Term Care: Support for Family Caregivers. Long-Term Financing Project. Washington, Georgetown University Press, 2004.

-15 Ory MG, Hoffman RR III, Yee JL, Tennstedt S, Schulz R: Prevalence and impact of caregiving: a detailed comparison between dementia and nondementia caregivers. Gerontologist 1999;39:177-185.

-16 Schulz R, O’Brien AT, Bookwala J, Fleissner K: Psychiatric and physical morbidity effects of dementia caregiving: prevalence, correlates, and causes. Gerontologist 1995;35:771-791.

-17 Ware JE, Kosinski M, Keller SD: A 12-Item Short-Form Health Survey: construction of scales and preliminary tests of reliability and validity. Med Care 1996;34:220-233.

-18 Ware JE Jr, Gandek B: Overview of the SF-36 Health Survey and the International Quality of Life Assessment (IQOLA) Project. J Clin Epidemiol 1998;51:903-912.

-19 Ware JE Jr, Sherbourne CD: The MOS 36-item short-form health survey (SF-36). I. Conceptual framework and item selection. Med Care 1992;30:473-483.

-20 Jenkinson C, Layte R: Development and testing of the UK SF-12 (short form health survey). J Health Serv Res Policy 1997;2:14-18.

-21 Kaufer DI, Cummings JL, Christine D, Bray T, Castellon S, Masterman D, MacMillan A, Ketchel P, DeKosky S: Assessing the impact of neuropsychiatric symptoms in Alzheimer's disease: the Neuropsychiatric Inventory Caregiver Distress Scale. J Am Geriatr Soc 1998;46:210-215.

- 22 Kaufer DI, Cummings JL, Ketchel P, Smith V, MacMillan A, Shelley T, Lopez OL, DeKosky ST: Validation of the NPI-Q, a brief clinical form of the Neuropsychiatric Inventory. J Neuropsychiatry Clin Neurosci 2000;12:233-239.

-23 Cummings JL, Mega M, Gray K, Rosenberg-Thompson S, Carusi DA, Gornbein J: The Neuropsychiatric Inventory: comprehensive assessment of psychopathology in dementia. Neurology 1994;44: 2308-2314. 
24 Cummings JL: The Neuropsychiatric Inventory: assessing psychopathology in dementia patients. Neurology 1997;48:S10-S16.

25 Tabachnick BG, Fidell LS: Using Multivariate Statistics, ed 5. Boston, Pearson Education, 2007.

26 Hulley SB, Cummings SR, Browner WS, Grady DG, Newman TB: Designing Clinical Research, ed 3. Philadelphia, Lippincott Williams \& Wilkins, 2007.

-27 De Vugt ME, Riedijk SR, Aalten P, Tibben A, van Swieten JC, Verhey FRJ: Impact of behavioral problems on spousal caregivers: a comparison between Alzheimer's disease and frontotemporal dementia. Dement Geriatr Cogn Disord 2006;22:35-41.

-28 Robert PH, Onyike CU, Leentjens AFG, Dujardin K, Aalten P, Starkstein S, Verhey FR, Yessavage J, Clement JP, Drapier D, Bayle F, Benoit M, Boyer P, Lorca PM, Thibaut F, Gauthier S, Grossberg G, Vellas B, Byrne J: Proposed diagnostic criteria for apathy in Alzheimer's disease and other neuropsychiatric disorders. Eur Psychiatry 2009;24:98-104.

29 Merrilees J, Dowling GA, Hubbard E, Mastick J, Ketelle R, Miller BL: Characterization of apathy in persons with frontotemporal dementia and the impact on family caregivers. Alzheimer Dis Assoc Disord 2012, E-pub ahead of print. 